\title{
Analytical Joint Processing Multi-Point Cooperation Performance in Rayleigh Fading
}

\author{
Dorra Ben Cheikh $^{\dagger \star}$, Jean-Marc Kelif ${ }^{\star}$, Marceau Coupechoux ${ }^{\dagger}$ and Philippe Godlewski ${ }^{\dagger}$ \\ * Orange Labs Issy-les-moulineaux, France, Email: \{dorra.bencheikh, jeanmarc.kelif\}@orange.com \\ $\dagger$ †ELECOM ParisTech \& CNRS LTCI, Paris, France, Email: \{bencheikh, coupecho, godlewski\}@enst.fr
}

\begin{abstract}
Coordinated multi-point (CoMP) is a very promising transmission scheme since it permits interference reduction and cell-edge throughput improvement. In this work, we study the performance of the joint processing multiple antenna multipoint cooperation using the maximum ratio transmission (MRT) technique. A closed form expression of the outage probability is derived for Rayleigh flat fading channel model considering pathloss and constant shadowing. Analytical and simulation results are compared.
\end{abstract}

Index Terms-CoMP, MRT, outage probability, multiple antenna, Rayleigh fading.

\section{INTRODUCTION}

Coordinated multi-point transmission is a new technique targeted to the LTE-Advanced (LTE-A) standard and promising better cellular performance. In the 3rd Generation Partnership Project (3GPP) LTE-A two main schemes were highlighted [1]: coordinated beamforming/scheduling (CoMPCBF) and joint processing (CoMP-JP). In the CoMP-CBF strategy, a user is served by only one base station (the master cell) and the surrounding BSs schedule users so as to generate the least interference possible to the user of the master cell. In the CoMP-JP strategy, coordinated BSs share information data to serve a user cooperatively. The CoMP-JP generates higher backhaul load since the cooperating BSs need to share user data, channel state information (CSI) and synchronization signals, while the CoMP-CBF needs to share only CSI and scheduling decisions. However, the JP strategy offers larger performance gain than the $\mathrm{CBF}[2,3]$. In this paper, we focus on CoMP-JP transmission. In [4], a measurement study showed that multicell cooperation attain larger mean capacity than an isolated cell when considering a sufficiently high capacity and low latency backbone. In [5], field trial was performed to confirm the throughput enhancement introduced by CoMP-JP strategy. In [6], a numerical study of different joint processing schemes showed the potential of this technique to enhance the overall system performance. In [7], the performance of the femtocell coordination strategy was studied for zero forcing (ZF) and maximum ratio transmission (MRT) schemes. Two power allocation algorithms were proposed and compared. In [4-7], authors performed simulation, measurement or field study but no theoretical studies were conducted. In [8], an analytical expression of the capacity outage probability was derived for an open-loop Alamoutilike CoMP downlink transmission in Rayleigh fading. The proposed SINR expression can only be achieved when using a distributed Alamouti for two cooperating BSs. In [9], an analytical study of a multi-cell multi-antenna cooperative MRT/MRC scheme was conducted. An analytical expression of the probability density function (PDF) of the signal-tointerference ratio (SIR) was derived considering path-loss, shadowing and Rayleigh fading. However, the authors resorted to many assumptions: a cell-edge user served in cooperation is at equal distances from the cooperative BSs, a Gamma distributed shadowing and a Poisson spatial distribution of interfering transmitters. Furthermore, there is a significant difference between simulation and theoretical results.

In this work, our main contribution is to perform an analytical study of a downlink multicell cooperation system using the MRT precoding technique. The MRT [10] is a transmission technique achieving maximum transmit diversity and maximizing the SNR. We propose an approximate outage probability expression of the downlink multiple antenna CoMPJP using the MRT precoding considering path-loss, constant shadowing and Rayleigh fading. Our analytical approach consolidates the numerical performance studies provided in the literature.

This paper is outlined as follows. In the next section, we introduce the system model. In section III, the outage probability expression is derived. In section IV, simulation results are presented and discussed. Concluding remarks are proposed in section $\mathrm{V}$.

The following notations are used: $(.)^{T}$ denotes the transpose conjugate operator, $\mathrm{E}[$.$] the expectation value and \operatorname{var}($.$) the$ variance.

\section{SySTEM MODEL}

Consider a downlink multicellular ( $B$ base stations) multiuser system ( $K$ active users) and consider multiple antenna BSs ( $M$ antennas) and single antenna user equipments. Let a cluster be a subset of BSs cooperating to serve a user. The clusters are disjoint. The selection algorithm of the BSs in a cluster is beyond the scope of this work, a possible simple criterion is the minimization of the distance depending pathloss. A cluster of BSs transmits to a single user per transmit time interval (TTI). BSs use the MRT to transmit their data. We assume coherent multicell transmission which needs a tight synchronization across transmitting BSs (like in [11]) that can be ensured using low-latency and high-capacity backhaul communication. The information data intended to a user are shared by all BSs in its cooperation cluster. CSI between 
the considered user and the cooperating BSs are estimated using feedback for the frequency division duplex (FDD) mode or uplink-downlink channel reciprocity for the time division duplex (TDD) mode. The cluster of BSs serving a user $k$ is denoted $B_{k}$. The signal received by a user $k$ is given by [12]:

$$
y_{k}=\sum_{b \in B_{k}} \sqrt{p_{b, k}} \mathbf{h}_{b, k} \mathbf{x}_{b, k}+\sum_{i=1, i \neq k}^{K} \sum_{j \in B_{i}} \sqrt{p_{j, k}} \mathbf{h}_{j, k} \mathbf{x}_{j, i}+n
$$

where $p_{b, k}$ is the power received by user $k$ from BS $b, \mathbf{h}_{b, k} \in$ $\mathbb{C}^{1 \times M}$ is the complex Gaussian channel between user $k$ and BS $b, n$ is the AWGN and $\mathbf{x}_{b, k} \in \mathbb{C}^{M \times 1}$ is the MRT data vector transmitted from BS $b$ to the user $k$ and is given by:

$$
\mathbf{x}_{b, k}=\frac{\mathbf{h}_{b, k}^{T}}{\left\|\mathbf{h}_{b, k}\right\|} s_{k}
$$

$s_{k} \in \mathbb{C}$ is the normalized information symbol intended to user $k$ from BS $b$. The received power $p_{b, k}$ includes path-loss and shadowing:

$$
p_{b, k}=P_{T} C d_{b, k}^{-\eta} 10^{\frac{\xi_{b, k}}{10}},
$$

where $P_{T}$ is the total transmit power of $\mathrm{BS} b, C$ is a constant, $d_{b, k}$ is the distance between the considered user and $\mathrm{BS} b, \eta$ is the path-loss exponent and $\xi_{b, k}$ is a Normal random variable with zero mean and standard deviation $\sigma$.

The output SINR perceived by a user $k$ is given by:

$$
\begin{aligned}
\gamma_{k} & =\frac{\left(\sum_{b \in B_{k}} \sqrt{p_{b, k}} \mathbf{h}_{b, k} \frac{\mathbf{h}_{b, k}^{T}}{\left\|\mathbf{h}_{b, k}\right\|}\right)^{2}}{\sum_{i=1, i \neq k}^{K}\left|\sum_{j \in B_{i}} \sqrt{p_{j, k}} \mathbf{h}_{j, k} \frac{\mathbf{h}_{j, i}^{T}}{\left\|\mathbf{h}_{j, i}\right\|}\right|^{2}+\sigma_{n}^{2}}, \\
= & \frac{\left(\sum_{b \in B_{k}} \sqrt{p_{b, k}}\left\|\mathbf{h}_{b, k}\right\|\right)^{2}}{\sum_{i=1, i \neq k}^{K}\left|\sum_{j \in B_{i}} \sqrt{p_{j, k}} \mathbf{h}_{j, k} \frac{\mathbf{h}_{j, i}^{T}}{\left\|\mathbf{h}_{j, i}\right\|}\right|^{2}+\sigma_{n}^{2}} .
\end{aligned}
$$

In dense urban interference limited system, the noise power can be neglected compared to the interference power, thus the SINR can be approximated as:

$$
\gamma_{k} \approx \frac{X}{Y}
$$

where

$$
\begin{aligned}
X & =\left(\sum_{b \in B_{k}} \sqrt{p_{b, k}}\left\|\mathbf{h}_{b, k}\right\|\right)^{2}, \\
Y & =\sum_{i=1, i \neq k}^{K}\left|\sum_{j \in B_{i}} \sqrt{p_{j, k}} \mathbf{h}_{j, k} \frac{\mathbf{h}_{j, i}^{T}}{\left\|\mathbf{h}_{j, i}\right\|}\right|^{2} .
\end{aligned}
$$

\section{Outage Probability}

The outage probability is an important QoS performance metric since it measures the probability of failure to satisfy a required threshold for a given service. It is defined as:

$$
P_{\text {out }}=P\left[\gamma_{k}<\gamma_{t h}\right] \approx P\left[\frac{X}{Y}<\gamma_{t h}\right],
$$

where $\gamma_{t h}$ is the SINR threshold value characterizing the considered service.

Let us derive the PDF of $X . X$ can be written as:

$$
X=U^{2}, \quad U=\sum_{b \in B_{k}} \sqrt{p_{b, k}}\left\|\mathbf{h}_{b, k}\right\|
$$

$U$ can also be written as:

$$
U=\sum_{b \in B_{k}} \sqrt{p_{b, k}} \sqrt{\sum_{i=1}^{M}\left|h_{b, k, i}\right|^{2}}
$$

To the best of our knowledge, there is no possible closed form expression for the PDF of $U$. We will hence use the central limit approximation for causal functions [13]. It permits to approximate the sum of positive independent and not necessarily identically distributed random variables by a Gamma distribution given by:

$$
f_{U}(u)=\frac{u^{\nu-1} e^{-\frac{u}{\theta}}}{\Gamma(\nu) \theta^{\nu}}
$$

where $\nu=\frac{\mathrm{E}[U]^{2}}{\operatorname{var}(U)}$ and $\theta=\frac{\operatorname{var}(U)}{\mathrm{E}[U]}$.

To derive $\nu$ and $\theta$, let us calculate the mean and the variance of $U, \mathrm{E}[U]$ can be derived as follows:

$$
\mathrm{E}[U]=\sum_{b \in B_{k}} \sqrt{p_{b, k}} \mathrm{E}\left[\sqrt{\sum_{i=1}^{M}\left|h_{b, k, i}\right|^{2}}\right] .
$$

Denoting $V=\sqrt{\sum_{i=1}^{M}\left|h_{b, k, i}\right|^{2}}$, it can be noticed, that $V$ is a square root of a Gamma distributed random variable and thus we can write the PDF of $V$ as:

$$
f_{V}(v)=\frac{2}{(M-1) !} v^{2 M-1} e^{-v^{2}}
$$

$\mathrm{E}[V]$ can be derived using (14) and is given by:

$$
\mathrm{E}[V]=\int_{0}^{\infty} \frac{2}{(M-1) !} v^{2 M} e^{-v^{2}} d v=\frac{(2 M-1) ! !}{2^{M}(M-1) !} \sqrt{\pi},
$$

where $(2 N+1) ! !=1 \times 3 \times 5 \ldots \times(2 N+1)$ and $(2 N) ! !=$ $2 \times 4 \times \ldots(2 N)$. From (13), $\mathrm{E}[U]$ is given by:

$$
\mathrm{E}[U]=\frac{(2 M-1) ! !}{2^{M}(M-1) !} \sqrt{\pi} \sum_{b \in B_{k}} \sqrt{p_{b, k}} .
$$

The variance of $U$ can be derived as follows:

$$
\begin{aligned}
\operatorname{var}(U) & =\mathrm{E}\left[\sum_{b \in B_{k}} \sqrt{p_{b, k}}\left\|\mathbf{h}_{b, k}\right\| \sum_{n \in B_{k}} \sqrt{p_{n, k}}\left\|\mathbf{h}_{n, k}\right\|\right] \\
& -\left(\mathrm{E}\left[\sum_{b \in B_{k}} \sqrt{p_{b, k}}\left\|\mathbf{h}_{b, k}\right\|\right]\right)^{2}, \\
& =\sum_{b \in B_{k}} p_{b, k} \mathrm{E}\left[\left\|\mathbf{h}_{b, k}\right\|^{2}\right]-\sum_{b \in B_{k}} p_{b, k} \mathrm{E}\left[\left\|\mathbf{h}_{b, k}\right\|\right]^{2} \\
& =\left(M-\pi\left(\frac{(2 M-1) ! !}{2^{M}(M-1) !}\right)^{2}\right) \sum_{b \in B_{k}} p_{b, k} .
\end{aligned}
$$


The parameters $\nu$ and $\theta$ are thus given by:

$$
\begin{aligned}
\nu & =\frac{(2 M-1) !^{2}}{M 2^{2 M}(M-1) !^{2}-\pi(2 M-1) ! !^{2}} \frac{\left(\sum_{b \in B_{k}} \sqrt{p_{b, k}}\right)^{2}}{\sum_{b \in B_{k}} p_{b, k}}, \\
\theta & =\frac{M 2^{2 M}(M-1) !^{2}-\pi(2 M-1) ! !^{2}}{2^{M}(M-1) !(2 M-1) ! ! \sqrt{\pi}} \frac{\sum_{b \in B_{k}} p_{b, k}}{\sum_{b \in B_{k}} \sqrt{p_{b, k}}}(19)
\end{aligned}
$$

Using the Gamma approximation of the PDF of $U$, we can derive the CDF of $X$ as follows:

$$
\begin{aligned}
F_{X}(v) & =\int_{0}^{\sqrt{v}} \frac{u^{\nu-1} e^{-\frac{u}{\theta}}}{\Gamma(\nu) \theta^{\nu}} d u \\
& =\frac{\gamma\left(\nu, \frac{\sqrt{v}}{\theta}\right)}{\Gamma(\nu)}
\end{aligned}
$$

where $\gamma(.,$.$) is the lower incomplete Gamma function and \Gamma($. is the Gamma function.

It is clear from (16) and (17) that adding new cooperating BSs improves the mean useful signal power but increases also its variability. We can also show that the mean and the variance are increasing with $M$.

Let us derive, now, the PDF of $Y$. We can write the expression (8) as:

$$
Y=\sum_{i=1, i \neq k}^{K}\left|\sum_{j \in B_{i}} \sqrt{p_{j, k}} g_{j, k, i}\right|^{2}
$$

where $g_{j, k, i}=\mathbf{h}_{j, k} \frac{\mathbf{h}_{j, i}^{T}}{\left\|\mathbf{h}_{j, i}\right\|}$. It was proven in [14], that, since the elements of $\mathbf{h}_{j, k}$ and $\mathbf{h}_{j, i}$ are zero-mean complex Gaussian random variables, $g_{j, k, i}$ is also complex Gaussian independent of $\mathbf{h}_{j, i}$. Let $c_{k, i}=\sum_{j \in B_{i}} \sqrt{p_{j, k}} g_{j, k, i}$, it is the sum of independent complex Gaussian random variables. $Y$ can be written as:

$$
Y=\sum_{i=1, i \neq k}^{K}\left|c_{k, i}\right|^{2}
$$

$\left\{c_{k, i}\right\}_{(i=1 \ldots K, i \neq k)}$ being independent zero-mean complex Gaussian elements with variances:

$$
\lambda_{i, k}=\operatorname{var}\left(c_{k, i}\right)=\sum_{j \in B_{i}} p_{j, k} .
$$

The PDF of $Y$ is, hence, given by [15]:

$$
f_{Y}(y)=\sum_{i=1, i \neq k}^{K} \frac{\Pi_{i}}{\lambda_{i, k}} \exp \left(-\frac{y}{\lambda_{i, k}}\right)
$$

where $\Pi_{i}=\prod_{p=1 . . K, p \neq k, p \neq i} \frac{\lambda_{p, k}}{\lambda_{p, k}-\lambda_{i, k}}$.

Some $\left\{\lambda_{p, k}\right\}_{p=1 . . K, p \neq k}$ may be equal. In this case, a very small number can be added to differentiate equal terms [15] and hence the distribution (25) is still valid and yields good results.

Having the PDF of $X$ and the PDF of $Y$, and since they are independent random variables, we derive the outage probability as follows:

$$
\begin{aligned}
P\left(\gamma_{k}<\gamma_{t h}\right)= & \int_{0}^{\infty} F_{X}\left(\gamma_{t h} y\right) f_{Y}(y) d y \\
= & \sum_{i=1, i \neq k}^{K} \frac{\Pi_{i}}{\lambda_{i, k} \Gamma(\nu)} \times \\
& \int_{0}^{\infty} \exp \left(-\frac{y}{\lambda_{i}}\right) \gamma\left(\nu, \frac{\sqrt{\gamma_{t h} y}}{\theta}\right) d y .
\end{aligned}
$$

The outage probability is given by [16]:

$$
P\left(\gamma_{k}<\gamma_{t h}\right)=\sum_{i=1, i \neq k}^{K} \Pi_{i}\left(\frac{\sqrt{\gamma_{t h} \lambda_{i, k}}}{2 \theta}\right)^{\nu} U\left(\frac{\nu}{2}, \frac{1}{2}, \frac{\gamma_{t h} \lambda_{i, k}}{4 \theta^{2}}\right)
$$

where $U(., .,$.$) is the confluent hypergeometric function of$ second kind. Expression (28) is a closed form approximation of the outage probability. It is a finite sum over the number of the active users in the network of easily computable elements allowing for rapid evaluation of the cooperative MISO system performance.

\section{Simulation Results}

Fig. 1 presents a comparison between simulated and theoretical outage probability. We consider a network of $19 \mathrm{BSs}$ equipped with 4 antennas (the central cell and two surrounding rings of BSs). We consider $N=3,4$ or 5 cooperating BSs and 5 interfering clusters of BSs so that $K=6$. A user is served by the $N$ BSs with smallest path-loss degradation. We first generate 19 realizations of the shadowing using a lognormal random variable with $\sigma=6 \mathrm{~dB}$ standard deviation. In each iteration of our Monte Carlo simulation, the SINR is calculated using these same realizations. All BSs emit a power of $20 \mathrm{~W}$. The cell radius is $R_{c}=500 \mathrm{~m}$ and the considered mobile station is at a distance $d=400 \mathrm{~m}$ from the central BS (celledge user). The path-loss exponent is $\eta=3.41$. In an urban environment and considering a frequency carrier $f_{0}=2 \mathrm{GHz}$, the path-loss constant $C=4.95 \times 10^{-4}$ [17]. The figure shows that there is a good match between simulations and analytical results. We can see that the approximation is all the more precise when the number of BSs is larger; however, it still holds even for a small number of cooperating BSs (3 or 4 $\mathrm{BSs}$ ). We can also notice the considerable gain of performance of the CoMP strategy over the non cooperative one.

In Fig. 2 we plot the outage probability of the SIR and of the SINR for $R_{c}=500 \mathrm{~m}$ and $R_{c}=1 \mathrm{Km}$. Thermal noise power is computed as $\sigma_{n}^{2}=N_{0} W$ where $N_{0}=-174 \mathrm{dBm} / \mathrm{Hz}$ is the noise density power and $W$ is the system bandwidth. Considering the same system parameters as for Fig. 1, it can be seen that for a dense urban environment where $R_{c} \leq 500 \mathrm{~m}$ and for a bandwidth of $W=20 \mathrm{MHz}$, the two curves are superposed, thus the influence of the noise power is negligible compared to the interference power. In this case the system is said interference limited. The difference between SIR and SINR outage probability becomes significant only for large cell ranges $\left(R_{c} \geq 1 \mathrm{Km}\right)$, large system bandwidths $(W \geq$ $10 \mathrm{MHz}$ ) and high SINR. On Fig. 2, we can note however 


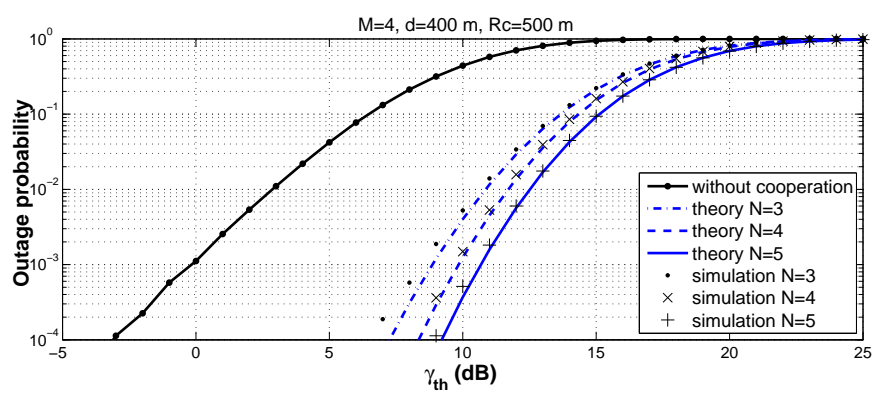

Fig. 1. Comparison between simulated and analytical results of the outage probability without CoMP and with CoMP MRT strategy for 3, 4 or 5 cooperating BSs.

that even for $R_{c}=1 \mathrm{Km}, W=20 \mathrm{MHz}$, our formula can be efficiently used in the low SINR region (for coverage studies for example).

In Fig. 3 we fix $N=3$ and varies $M=1,2$ or 4 . Again, we notice that our formula provides a good approximation of the outage probability. As an expected result, we can observe that increasing the number of antenna per BS enhances the system performance and that the improvement is decreasing with $M$. This is a classical result related to the diversity gain brought by multiple antenna at the BSs.
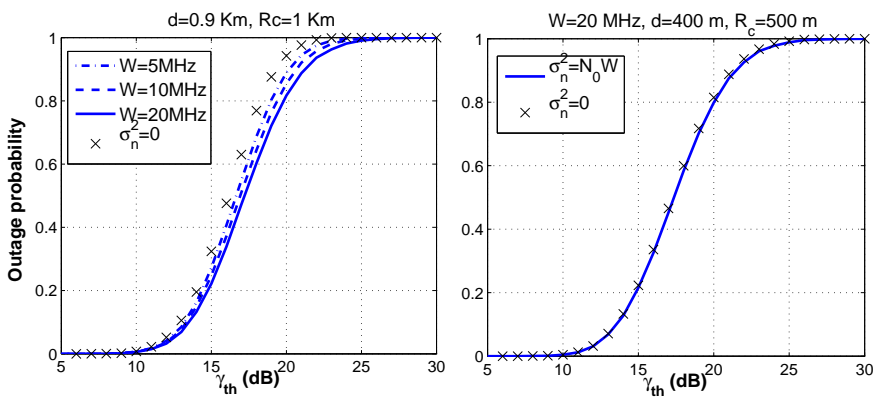

Fig. 2. Impact of the noise power on the outage probability.

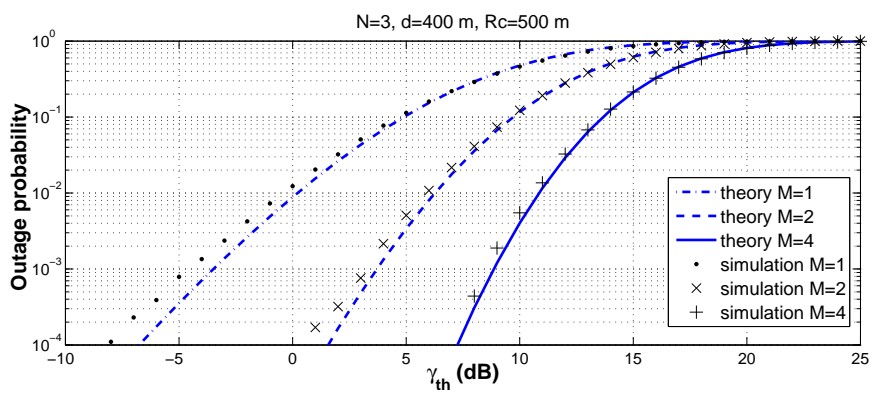

Fig. 3. Outage probability versus SINR threshold for a downlink multicellular system using CoMP and $M=1,2,4$ antennas per BS.

\section{CONCLUSION}

In this paper, the performance of the joint processing maximum ratio transmission technique was studied in terms of outage probability. A closed-form expression has been derived using a Gamma approximation of useful power PDF and an exact interference power PDF. Simulation validates the derived outage probability expression. Our model is definitly optimistic since we assume perfect CSI at the transmitter. A more realistic approach should take into consideration the imperfectness of the CSI or consider open loop diversity achieving techniquesm, e.g., space time coding.

\section{REFERENCES}

[1] 3GPP TR 36.814, "Evolved Universal Terrestrial Radio Access (E-UTRA); Further advancements for E-UTRA physical layer aspects," R9 V9.0.0, Mar. 2009.

[2] J. Li, E. Lu, and I.-T. Lu, "Performance Benchmark for Network MIMO Systems: A Unified Approach for MMSE Transceiver Design and Performance Analysis," in Proc. IEEE GLOBECOM, pp. 1-6, 2010.

[3] Y. Rui, M. Li, P. Chengo, Y. Luo, and A. Guo, "Achievable Rates of Coordinated Multi-Point Transmission Schemes under Imperfect CSI," in Proc. IEEE ICC, pp. 1-6, 2011.

[4] V. Jungnickel and al., "Capacity Measurements in a Cooperative MIMO Network," IEEE Trans. Veh. Technol., vol. 58, pp. 23922405, Jun. 2009.

[5] P. Marsch, M. Grieger, and G. Fettweis, "Large Scale Field Trial Results on Different Uplink Coordinated Multi-Point (CoMP) Concepts in an Urban Environment," in Proc. IEEE WCNC, pp. 1858-1863, 2011.

[6] H. Zhang, H. Dai, and Q. Zhou, "Base Station Cooperation for Multiuser MIMO: Joint Transmission and BS Selection," in Proc. Conf. Inform. Sciences and Sys., 2004.

[7] S. Ben Halima, M. Helard, and D. Phan-Huy, "New Coordination and Resource Allocation Schemes for Uniform Rate in Femtocell Networks," in Proc. IEEE VTC-Spring, pp. 1-5, 2011.

[8] V. Garcia, N. Lebedev, and J.-M. Gorce, "Capacity Outage Probability for Multi-Cell Processing Under Rayleigh Fading," IEEE Commun. Lett., vol. 15, pp. 801-803, Aug. 2011.

[9] X. Ge, K. Huang, C.-X. Wang, X. Hong, and X. Yang, "Capacity Analysis of a Multi-Cell Multi-Antenna Cooperative Cellular Network with Co-Channel Interference," IEEE Trans. Wireless Commun., vol. 10, pp. 3298-3309, Oct. 2011.

[10] T. K. Y. Lo, "Maximum Ratio Transmission," IEEE Trans. Commun., vol. 47, pp. 1458-1461, Oct. 1999.

[11] E. Bjornson, R. Zakhour, D. Gesbert, and B. Ottersten, "Cooperative Multicell Precoding: Rate Region Characterization and Distributed Strategies With Instantaneous and Statistical CSI," IEEE Trans. Wireless Commun., vol. 58, pp. 4298-4310, Aug. 2010.

[12] A. Tolli, H. Pennanen, and P. Komulainen, "On the Value of Coherent and Coordinated Multi-Cell Transmission," in Proc. IEEE ICC Workshops, 2009.

[13] A. Papoulis, The Fourier Integral and Its Applications. New York, NY: McGraw-Hill, 1962.

[14] A. Shah and A. Haimovich, "Performance Analysis of Maximum Ratio Combining and Comparison with Optimum Combining for Mobile Radio Communications with Cochannel Interference," IEEE Tran. Veh. Technol., vol. 49, pp. 1454-1463, Jul. 2000.

[15] R. Visoz and E. Bejjani, "Matched Filter Bound for Multichannel Diversity Over Frequency-Selective Rayleigh-Fading Mobile Channels," IEEE Trans. Veh. Technol., vol. 49, pp. 1832 1844, Sep. 2000.

[16] I. Gradshteyn and I. Ryzhik, Table of integrals series and products. Moscow: Academic Press, 1963.

[17] J.-M. Kelif, M. Coupechoux, and F. Marache, "Limiting Power Transmission of Green Cellular Networks: Impact on Coverage and Capacity," in Proc. IEEE ICC, pp. 1-6, 2010. 